\title{
Review Article \\ Dancing bees speak in a code-A Review
}

Received: 20 July 2020

Accepted: 23 September 2020

Online: 24 September 2020

Authors:

A. Singla

Department of Entomology, Punjab Agricultural

University, Ludhiana, Punjab, India

$\bigotimes$ akankshasingla684@gmail.com

Emer Life Sci Res (2020) 6(2): 44-53

E-ISSN: 2395-6658

P-ISSN: 2395-664X

DOI: https://doi.org/10.31783/elsr.2020.624453

\section{Akanksha Singla}

\begin{abstract}
Honeybee foragers dance to communicate the quantity, quality, direction, and spatial location of food and other resources to their nestmates. This remarkable communication system has long served as an important model system for studying the mechanisms and evolution of this complex behavior. I provide a broad overview of the research done on dance communication, earlier theories, and their controversies and solution. Specific issues concentrated in this review are as follows: (a) different types of dances (b) measurement of distance and direction (c) How bees perform dance in dark hive? Various experiments have confirmed that bees perform different types of dance depending upon their specific function. However, detailed information about other types of dances is still lacking which if worked on will help us in solving various queries and would help us in better understanding the significance of different types of dances performed by honeybees in and outside the hive.
\end{abstract}

Keywords communication, dance, distance, direction, honeybee

\section{Introduction}

Insects, especially bees, play a key role in the pollination of the majority of the world's fruit, seed and vegetable crops. Both the crop yield and quality [1-3] are known to receive benefits from these ecosystem services. Although wild insects can also be effective crop pollinators [4], the economic value added by honeybee spp., Apis mellifera is perhaps equal to that of all other bee species in combination. However, the question is how these bees recruit other bees to reach the food source. The honeybee "dance language" which was discovered by Karl von Frisch and thus awarded with the 1973 Nobel Prize in Physiology or Medicine is used by honeybees to share the information regarding the location of foraging [5]. This distinctive behavior has been used to explore abundant aspects of honeybee foraging ecology [6-8]. Although crop pollination is a well-researched topic, to our knowledge, the language of dance has never previously been used to determine where honeybees from colonies amongst a crop under commercial pollination are foraging [9]. Maximizing the potential of honeybees is likely to be of increasing importance to the global food supply, given that the demand for pollination services is rising at a greater rate than hive numbers [10-11].

\section{Early theories}

Aristotle, more than 2000 years ago, observed that when a dish of honey is kept near the hive, it usually took hours or even days for a worker honeybee to locate it. However, once a single bee found the food, the arrival of other bees soon started. The forager must in some way have recruited the other bees to reach the food source. Aristotle thought that the forager was simply 
followed by the forager bees for the food. Maeterlinck [12] tested this justification by allowing a forager bee to locate food, return to the hive, and was caught while on its way back out of the hive. He observed that although no forager was available for the recruits to be followed to the food source, even then, some of them found the food. He speculated that forager must be using some "tactile language or magnetic induction" to guide the recruits to reach the exact location of the food. Frisch [13] also did a series of experiments by training European honeybee, A. mellifera to feeders and initially believed that bees use flower scents or other odors to find the food source. However, he later started paying close attention to the dances performed by the returning foragers. Finally, by 1947, he reported that bees use a symbolic communication - a 'language' or 'secret sign' to provide distance and direction information which was used by the forager bees.

\section{Controversy and solution}

Karl von Frisch in spite of winning the Nobel prize in 1973 for his discovery of dance language, faced a major challenge to his theory by two scientists, Adrian Wenner and Patrick Wells from California. They claimed that decoding of the dance language by Frisch was a mere illusion and bees locate food discovered by the foragers using the scent left by the new discoverer on the way to and at the location of the food. Frisch et al., [14] trained the foragers of the experimental hive (a colony of dark-colored bees) to the forager station by providing a richly scented, concentrated sugar solution. On the other hand, foragers of the control hive (a colony of light-colored bees) were trained in the same way to three recruit stations. Recruits arriving at all the stations were killed after being captured. When the experimental hive was opened alone, recruits as expected arrived at or near the forager station. While, when the control hive foragers were set free to visit the recruit stations, providing bee, hive, scent gland odor as well as the sight of bees at each station, preference disappeared. The experimental hive showed the same distribution as control hive recruits. Thereby, concluding that dance information was not used for locating the food source. He again attempted the same experiment by controlling the forager generated visual and odor cues which according to him might were deviating the results. But, he again arrived at the same conclusion and thus confirmed that information in dance was not used by bees.

Further, Frisch et al., [14] performed the same experiment and the similar outcomes were recovered. However, an opposing argument that bees possess a very well developed sense of smell could not be ignored. They are indeed capable of finding food discovered under some situations by their sisters using the scent of the discoverer on the way to and at the location of the food. But, the query was not whether bees can ever trace the food source successfully by means of only odor cues left by the discoverer. The matter of interest was: Can bees communicate effectively using dance language without the requirement of scent marks of the discoverer along the way to and at the target location? This was possible only on retaining barely the information given by the discoverer and eliminating the scent marks of the dancer. And this was accomplished in an inventive way by Gould [15]. He made the bees scout "lie" and dance for a food source that they had never visited. Ocelli or simple eyes which perceive the light intensity was covered for some forager bees, making them less sensitive to light and preventing them from reorienting their dances. Also, the bright light was placed over the hive leading to the orientation of their dances according to that light instead of gravity. The main idea behind this experiment was to misdirect the bees with uncovered ocelli to test the existence of a true language. The experiment revealed that bees arrived at a location specified by the dance and did not arrive at the odor-marked forager station. Thus, subsiding the controversy that dance language communicates information about the location and quality of food source and confirmed that odor probably affected the speed and accuracy of the recruits.

Recently, Landgraf et al., [16] justified the findings of Gould [15] using the honeybee robot, RoboBee that elicited the waggle dance pattern for flight vector chosen by the experimenter and even generated multiple cues contained in the biological dance pattern and natural dance following behavior in live bees. After following the dancing robot, the flight trajectory of hive leaving bees were tracked via harmonic radar and it was proved that bees use information obtained from the dance of the robotic bee to adjust their flight path. This was the first report on successful dance following and subsequent flight performance of bees recruited by a biometric robot. 


\section{Types of dances}

The recruitment of foragers for searching the food source starts when a scout bee loaded with abundant food from a newly discovered source returns to the hive. She regurgitates for 30-45 seconds and distributes the sample to bees waiting in the hive. Once her generosity has gathered enough audience, the dance begins. There are 3 broad types of bee dance: round dance, tail-wagging or waggle dance, and a transitional form of dance known as the sickle dance [17]. However, other types of dances are also reported by Grad [18] which are mentioned as: Shake dance, Tremble dance, Whir dance, grooming dance, massage dance, Alarm, and joy dance. The nature of dance performed is related to the distance to the source of food. Simple round dance is performed when the food source is near the colony. At bigger distances, a sickle dance is performed. At last, a waggle dance is performed at the farthest distances from the nest.

\section{Details of dance performed Round dance}

If a foraging honeybee (A. mellifera) locates a profitable food source in the immediate vicinity of the hive, she performs a round dance to communicate its location [19]. The forager bee moves over the comb in close circles, moving in the clockwise and anticlockwise direction [20]. Originally, Frisch supposed that round dance shared the information about a nectar resource, whereas the waggle dance communicated a pollen resource [21]. This misinterpretation between two types of dances arose from Frisch's training of bees to nectar feeders located near the hive, whereas pollen foragers exploited natural resources typically located at a greater distance from the hive. Later, findings by Frisch [22-23] confirmed that round dance was used for the nearby sources and does give an indication about the distance but no detailed information is given about direction and the type of food.

\section{Sickle dance}

When the food sources are located between 50 to 150 meters away from the hive, bees are recruited using sickle dance. This kind of dance is a figure of eight lacking the waggle phase in the middle. The form of this dance is crescent-shaped, regarded as a transitional dance between a round dance and a figure-eight waggle dance. As compared to the round dance indicating no direction to the food source, sickle dance communicates both the direction as well as the distance to the nesting site [19].

\section{Waggle dance}

The waggle dance encodes the most complex information of all the dances. A waggle dance consists of repeated runs of one to 100 or more circuits, each of which includes two phases: the waggle phase and the return phase. A worker bee's waggle dance involves running through a small figure-eight pattern: a waggle run (waggle phase) followed by a turn to the right to circle back to the starting point (return phase), another waggle run, followed by a turn and circle to the left, and so on in a regular alternation between right and left turns after waggle runs [24-25]. Through this dance, successful foragers share information with other members of the colony related to the distance and direction to flowers yielding nectar, pollen, water, and tree resin sources, or to new nest-site locations [26-30].

Frisch (1974) [20] also determined that the vital distance required for switching between the round and the waggle dance exists at 50 meters away from the hive. The scent attached to the forager bee's body communicates the type of food source to the follower bees. However, to guide the follower bees to the said food source, the scent of the food source alone was not observed to be ample information. Various other mechanisms were also reported to be used to communicate information regarding direction and distance to the food [19] which are listed below

\section{(a) Acoustic Signals}

Acoustic signals are produced as a part of the performance of the dance. As the sounds produced by this dance are airborne, they also have sufficient potency to excite Johnston's organs in the antenna of the follower bees to which the forager is communicating. Kirchner et al., [32] observed that the recruited bees 
extract information about the direction of the food source from the acoustic field that the forager produces. The direction was assessed through the sound information released by the forager bee, as well as, through the air currents accompanying the sound of dance. They also confirmed that these acoustic signals were also used to communicate distance. The time duration for the transmission of the signal was directly proportional to the distance to the food source. Thereby, the train of sound pulses produced by the forager bee, known as the dance sound, increases relative to the distance to the food source.

\section{(b) Sun's position}

The waggle dance also uses the sun's position to designate the direction of the food source [32]. On the vertical surface of the honeycomb, the forager expresses the angle between the position of the sun and the path to the food source through an angular deflection from perfect vertical [20]. Honeybees utilize both the situation of the sun and the polarization patterns of a blue sky to converse the way to the food source. Backing for this hypothesis rests in the perception that honeybees can in any case perceive the sun's position when it is masked by a cloud or a mountain, for example, Frisch in his trial affirmed that honeybees can perceive the everyday activities of the sun and can utilize the sun as a compass by ascertaining the hour of the day.

The edge (as displaced from vertical in A. mellifera) and the number of waggles in the waggling run of the move interpret the direction and distance, respectively, to the food source at which the dancer had most lately foraged. This dance is also reported to resume or reactivate the work of the experienced foragers which was interrupted. This statement was confirmed by the working of Biesmeijer and Seeley [31] who observed the extent up to which worker honeybees use waggle dance to extract information about foraging location. They concluded waggle dance used for food discovery accounted to $12-25 \%$ of all connections with dancers ( $9 \%$ by beginners and $3-16 \%$ by experienced foragers) whereas dance followed for reactivation and confirmation accounted for $75-88 \%$ (26\% for reactivation and 49-62\% for confirmation). They further confirmed that foragers don not use waggle dance only to start a new work or to find unfamiliar sources but honeybees also make widespread use of the waggle dance to resume work at old, familiar food sources. Grüter and Farina [32] also established that dance language is just one element of the waggle dance. The information obtained from it about the location is only used in case the information about food sources received directly from the environment is not available, i.e., food is of poor quality or the source of the food is unprofitable.

Waggle dance was also proved to be highly advantageous in disseminating information regarding the profitability of the food source located by the forager and the profitability is determined by the energetic value of the forager [33]. The quality and quantity of the food source determine the liveliness of the dance [20]. Further, Waddington and Kirchner [34] supported the explanation by stating that honeybees communicate information regarding the profitability of a food source through rate and number of reversals; and the duration of the dance. If the nectar source is of excellent quality, nearly all foragers will dance enthusiastically during each length of time they return from foraging. However, food sources of lower quality will produce fewer, shorter, and less vigorous dances, thereby recruiting fewer new foragers.

However, possible costs like, distance from the hive and nectar flow rate could be associated with the food source, thereby lowering the profitability of the source. Also, forager's rating criteria of profitability is highly dependent upon the quality of the food source formerly encountered. Afik et al., [33] reported that if the forager bee had recently encountered a food source of greater energetic value and low cost, she will then rate the present food to be less profitable. Thus, the forager bee integrates the costs and benefits associated with each potential food source and communicates their subjective profitability through their dance performances. However, the forager bee only shares information about the food source if it crosses a certain concentration threshold for dancing. Bypassing this threshold, it is concluded that there are a sufficient amount of benefits coupled with the food source to justify the spending of energy required for the performance of dance [35]. 


\section{Measurement of distance and direction}

The waggle dance is used by the forager bees for decoding the dance language and predicting the distance and direction to the food source. Waggle runs are performed over 100 times and the distance is correlated to the duration of the run while the information about the direction to the food is extracted from the angle of the waggle run relative to the vertical which provides the angle of the swarm in relation to the solar azimuth [36]. But how these bees gain the information delivered in the dance? Various mechanisms providing information about the direction of the food are well understood. The direction can be determined using multiple cues like landmarks [37-38]; the position of the sun, polarization pattern of skylight [19], and probably even magnetic fields [39]. Depending upon the environmental conditions, bees may prefer one mechanism over the other. But the mechanism(s) by which foragers find out or calculate the distance was not clear earlier as Frish during 1945-1947 conducted a series of experiments and proposed 'energy hypothesis' which stated that energy expenditure during the trip was the basis for the estimation of the distance. However, Esch and Burns [40] studied the flight behavior of the forager and projected 'optical flow hypothesis' in which it was stated that bees estimate the distance based on the speed of retinal image motion perceived from the ground. Srinivasan et al., [41] also unrevealed various ways in which bees use cues for locating food source using optic flow. They confirmed that bees possess a visually driven 'odometer' that is robust to the changes in wind load and expenditure of energy. And the distance to the resource is calculated by combining the apparent motion of the visual world experienced en route. The same results were recovered from the studies of Si et al., [42] where they found that the measurement of the distance is done using visually-driven odometer which is unaffected by the variations in the texture of the surroundings through which the bees fly. Dacke and Srinivasan [43] also performed similar experiments and observed that bees integrate the motion of the image and the distance is determined by calculating the extent to which the image of the environment moves in the eye of the bees as they fly towards their resource. However, in 2008, Dacke and Srinivasan [43] concluded that there are two types of odometer which help in locating the food source, that is, 'community' odometer which is used to provide information using dance to the nestmates and a 'personal' odometer that is used by an experienced forager to return to the already known food source. Meanwhile, Grüter et al., [44] studied the most debated issue about the relative use of waggle dance (social information) versus route memories (private information) in locating the resource. They tested the hypothesis that dance language should be given due importance when the low benefit is available from private information. And they confirmed that the foragers increase the use of the information provided by the waggle dance when the private information yields them nothing.

\section{Other dances}

Besides round and waggle dances performed by the honeybees, several other dance forms are also presented, which are listed below [18]

\section{Grooming dance}

It is performed to ask another bee for grooming. Here, the bee stamps her feet and shakes her body. Pettis and Pankiw [45] recorded the role of grooming behavior in reducing the infestation of the parasitic mite, Acarapis woodi. Grooming behaviors included allogrooming and self-grooming. Hives monitored for $24 \mathrm{~h}$ revealed that dancing increased significantly at night while allogrooming decreased. In other experiments, they marked the young bees and introduced it into three hives with 0,50 , and $70 \%$ tracheal mite infestation. Thus, concluding that grooming dances increased significantly with an increase in infestation to the mite. Guzman-Novoa et al., [46] also tested the relationship between the infestation level of the parasitic mite, $V$. destructor in the honeybee, A. mellifera colonies, rate of mortality of fallen mites, and the intensity with which mites of different genotypes do auto grooming to remove mites from their bodies. They were likewise looked at the individual level for the power of grooming and mite expulsion achievement. Honeybees from the "resistant" settlements had lower mite population rate (up to 15 fold) and higher rates of harmed parasites (up to 9 fold) than honeybees from the "susceptible" genotypes. At the individual level, honeybees from the "resistant" genotypes performed essentially more occasions of serious grooming 
(up to 4 fold), and a fundamentally higher number of mites were removed from the body of honeybee by exceptional grooming than by light grooming (up to 7 fold) in all genotypes. The aftereffects of this examination firmly recommended that grooming behavior and the intensity with which honeybees perform it is a significant part of the opposition of some honeybee genotypes to the development of Varroa mite population.

According to Pritchard [47], grooming dance is categorized into two types: auto grooming/selfgrooming and inter-bee grooming/allogrooming. Early observations on the Eastern honeybee, A. cerana, recorded auto grooming, one-on-one, and social allogrooming, all of which resulted in death, visibly utilated varroa mites falling to the hive floor. Similar behavior was sought in the Western honeybee, $A$. mellifera, with variant observations for the different subspecies. Most depictions relate to A. m. carnica, some to A. m. ligustica, however with one prominent special case, practically none to A. m. mellifera. The most amazing discoveries are from "Africanized" honeybees, which give probably the best instances of natural, dependable resistance to varroa mites in A. mellifera, albeit even a portion of these is disputable.

The speed of both auto grooming and allogrooming reactions is commonly answered to be much slower in A. mellifera than in A. cerana, and the recurrence and level of harm to mites were likewise lower. Later, Land and Selley [48] video recorded the bees producing grooming invitation dance which stood stationary and vibrated their whole body from side to side with a frequency of $4.2+0.2 \mathrm{~Hz}$ for $9.3+1.0$ second. They observed that the bees that were puffed with chalk dust onto the bases of the wings produced significantly more grooming invitation dances as compared to the control bees that received only puffs of air. Thus, confirming that the major factor which triggered the grooming dance was the accumulation of small particles at the base of the wing.

\section{Joy dance}

The main purpose of this dance is to celebrate, for example, when a new queen emerges from her cell. In this dance, the bee places her front legs on one another and pulses her abdomen up and down.

\section{Massage dance}

Massage dance is performed to request a massage. In this dance form, the honeybee moves her head in a specific edge. Other honeybees react by pulling her leg joints and contacting her sides.

\section{Alarm dance}

Alarm dance warn others of the danger when a food source is contaminated. In this dance, the bee vibrates vigorously and runs in a zigzag, spiral motion .

\section{Tremble dance}

The main purpose of tremble dance is to inform other bees that a large load of nectar has arrived in the hive for processing. In this dance, the honeybee strolls comfortably and squirms its legs, making its body quiver and tremble. At first, Seeley [49] was the first person who clarified the message and the significance of a tremble move for the working drones working inside and outside the hive.

They explained that the message of the tremble dance as "I have visited a rich nectar source worthy of great exploitation, but already we have more nectar coming into the hive than we can handle" However, its explained meaning for the bees working inside the hive was interpreted as "I should switch to the task of processing nectar" while its meaning for the worker bees outside the hive was "I should refrain from recruiting additional foragers to any nectar source''. Hence, it was justified that the tremble dance functioned as a mechanism for keeping a match between the colony's nectar processing and the nectar influx rate. Further, Thom [50] performed an experiment to check if long unloading of nectar delay caused tremble dance and verified that the crowding of nectar foragers at the food source increased the probability of tremble dancing but was not caused due to long unloading delays, and the tremble dancers that foraged at natural food sources were often had short unloading delays. Moreover, when the cause of the tremble dance was not a low supply of nectar receiver bees, the tremble dance may have a function in addition to the recruitment of nectar receiver bees. Later, Seeley et al., [51] also reported the count of bees engaged in 
nectar reception before and after the stimuli of producing tremble dance was provided. They reported that in two trials, the number of nectar receivers rose from $17 \%$ of the colony's population before tremble dancing to $30-50 \%$ of the population after the dancing. They also investigated the age of additional nectar receivers, before and after the period of intense tremble dancing, and found that none of the bees were old bees who have recruited the task of nectar reception, however, most were middle-aged bees, and some were even young bees.

\section{Shake dance}

Its purpose is to inform house bees to help the foragers collect nectar from a, particularly rich source. In this dance, the forager bees shudder in front of one house bee at a time, to notify up to 20 per minute [25].

\section{Whir dance}

This kind of dance motivates the colony to leave the hive and swarm. In this dance, the bee runs in zigzags, whirs its wings, and shakes its body [25].

\section{How bees follow dance in dark hive?}

Honeybee species like A. florea (dwarf bee), A. dorsata (rock bee) perform dances on the upper outdoor face of the hive, while other hive bees as A. mellifera and A. cerana carry out dances in the hive. Honeybees periodically shake their bodies from left to right with their wings while performing dances. The former bee species conduct dances in the open and are visually conspicuous and the latter bee species could not utilize the visual features as they dance inside the hive where it is too dark. Some signals that may help in the communication of hive dancer with recruits were suggested by several researchers [52-53]. They recommended that various chemicals were produced and released by dancer bees which activate follower foraging. Floral odors were also thought to serve as a cue that allows followers to find the source of food. However, as several different odors prevail in the hive because of the storage of food and the activities of nestmates, it was still unclear if followers were able to utilize the cues of odor to find dancers in the hives. Several studies were carried out by different scientists [54-55] and they stated that during the waggle dance, honeybees buzz their wings, thereby, emitting a burst of sounds. These sounds consist of pulses, each pulse having a carrier frequency of $250-300 \mathrm{~Hz}$, and a pulse duration of $20 \mathrm{msec}$. Previous experiments conducted by Towne and Kirchner [56]; Kirchner et al., [57]; Dreller and Kirchner [58] established that for tuning themselves into this frequency, honeybees use Johnston's organ in antennae. Kirchner et al., [57] also found that after honeybees associated a $265 \mathrm{~Hz}$ tone stimulus with a reward following operant conditioning, they were able to localize the sound source and discriminate the dance frequency from a different frequency in a Y-maze. However, dance communication is not the only hive-related activity that includes the vibration of the wing. Other events like hive cooling also produce wing vibrations that emit sounds that are alike in the frequency of sounds produced by dance. Afterward, in the investigations of comb vibrations, signal intensification by the stage inversion phenomenon brought about by rehashed waggle runs was proposed to be significant in pulling in dance followers in a boisterous environment [59]. These vibration signals could be sensed by proprioceptors located in the bee's legs that respond in the range of $200-1000 \mathrm{~Hz}$ [60]. A study by Hasegawa and Ikeno [61] also investigated whether dance followers can discriminate dance sounds from other activities arising from wing vibrations. Honeybees were connected to a force meter to analyze their orienting reactions caused distinctly by sounds, and not by smell or vibrations detected with their legs.

Their results showed that honeybees were able to localize sounds with carrier frequencies of both $250 \mathrm{~Hz}$ and $265 \mathrm{~Hz}$ and were not able to distinguish between flight and repeated pulse sounds produced by the waggle dances. The trial utilizing distinctive musical dance sounds indicated that honeybees had the option to distinguish cadenced sounds inside a specific frequency range. They suggested that dancers can attract followers even in their noisy environments using frequency and rhythmic components of dance sounds. 


\section{Conclusion}

Although, dance language does not help bee in saving the time of recruits these different types of dances allow recruits to monopolize the rewarding food sources and exploiting them before they get discovered by the other colonies of the honeybees. Even, as the dance language provides detailed information on the quantity, quality, and direction of the food source, more research is needed to be done to better understand the other useful information provided by various kinds of dances in the form of codes. Thus, it could be justified that the dance language is the only means of providing data regarding the profitability, abundance, and other qualities of the source.

\section{References}

[1] M. Chagnon, J. Gingras and D. DeOliveira (1993). Complementary aspects of strawberry pollination by honey bees and indigenous bees (Hymenoptera). J. Econ. Entomol., 86: 416-420.

[2] D. Kleijn, R. Winfree, I. Bartomeus, L. G. Carvalheiro, M. Henry, R. Isaacs and A.-M. Klein et al., (2015). Delivery of crop pollination services is an insufficient argument for wild pollinator conservation. Nat. Commun., 6: 7414. doi: 10.1038/ncomms8414

[3] R. Bommarco, L. Marini and B. E. Vaissiere (2012). Insect pollination enhances seed yield, quality, and market value in oilseed rape. Oecologia, 169: 1025-1032.

[4] L. A. Garibaldi, I. Steffan-Dewenter, R. Winfree, M. A. Aizen, R. Bommarco, S. A. Cunningham and C. Kremen et al., (2013). Wild pollinators enhance fruit set of crops regardless of honey bee abundance. Science, 339: 1608-1611.

[5] T. D. Seeley (1995). The Wisdom of the hive: The social physiology of honey bee colonies. Harvard University Press.

[6] P. K. Visscher and T. D. Seeley (1982). Foraging strategy of honeybee colonies in a temperate deciduous forest. Ecology, 63: 1790-1801.

[7] M. Beekman, F. L. W. Ratnieks (2000). Long-range foraging by the honey bee, Apis mellifera L. Funct. Ecol., 14: 490-496.

[8] M. J. Couvillon, R. Schürch and F. L. W. Ratnieks (2014). Waggle dance distances as integrative indicators of seasonal foraging challenges. PLoS ONE, 9: e93495. doi:10.1371/journal.pone.0093495

[9] J. B. Free (1993). Insect Pollination of Crops. Academic Press, London.

[10] M. A. Aizen and L. D. Harder (2009). The global stock of domesticated honey bees is growing slower than agricultural demand for pollination. Curr. Biol., 19: 915-918.

[11] T. D. Breeze, B. E. Vaissière, R. Bommarco, T. Petanidou, N. Seraphides, L. Kozák, J. Scheper and J. C. Biesmeijer et al., (2014). Agricultural policies exacerbate honeybee pollination service supply demand mismatches across Europe. PLOS ONE, 9: e91459. doi: 10.1371/journal.pone.0091459

[12] M. Maeterlinck (1901). The life of the bee. New York: Dodd, Mead and Company.

[13] K .V. Frisch (1919). Uber den Gerusin der Bienen und seine blütenbiologische. Bedeutung. Zool. Jahrb., 97: $1-238$.

[14] K. V. Frisch, A. M. Wenner and D. L. Johnson (1967). Honey bees: do they use the direction and distance information provided by their dancers? Science, 158: 1072-1076.

[15] J. L. Gould (1975). Honey bee recruitment: the dance language controversy. Science, 189: 685-693.

[16] T. Landgraf, D. Bierbach, A. Kirbach, R. Cusing, M. Oertel, K. Lehmann and U. Greggers (2018). Dancing honey bee robot elicits dance-following and recruits foragers. arXiv preprint arXiv:1803.07126

[17] K. V. Frisch (1923). Über die "Sprache" der Bienen, eine tierpsychologische Untersuchung. Zool. Jahrb. Abt. Allg. Zool. Physiol., 40: 1-186.

[18] P. Grad (2010). How do bees communicate? They dance bee dances. https://bigislandbees.com/blogs/bee-blog/14137357-bee-dances

[19] W. Kirchner, M. Lindauer and A. Michelsen (1988). Honeybee dance communication. Naturwissenschaften, 75: 629-630.

[20] K .V. Frisch (1974). Decoding the language of the bee. Science, 185: 663-668. 
[21] T. Munz (2005). The bee battles: Karl von Frisch, AdrianWenner and the honey bee dance language controversy. J. Hist. Biol., 38: 535-570.

[22] K. V. Frisch (1946). Die Ta“nze der Bienen. O“sterr. Zool. Z., 1: 1-148

[23] K .V. Frisch (1967). The dance language and orientation of bees. Harvard University Press, Cambridge,

[24] C. Thom, D. C. Gilley, J. Hooper and H. E. Esch (2007). The scent of the waggle dance. PLoS Biol., 5: e228. doi:10.1371/journal.pbio.0050228

[25] T. D. Seeley, A. S. Mikheyev and G. J. Pagano (2000). Dancing bees tune both duration and rate of waggle run production in relation to nectar source profitability. J. Comp. Physiol., 186: 813-819.

[26] J. Riley, U. Greggers, A. D. Smith, D. R. Reynolds and R. Menzel (2005). The flight paths of honeybees recruited by the waggle dance. Nature, 435: 205-207.

[27] T. D. Seeley, P. K. Visscher and K. M. Passino (2006). Group decision making in honey bee swarms, American Scientist, 94: 220-229.

[28] M. Lindauer (1955). Schwarmbienen auf Wohnungssuche. Zeitschrift für vergleichende Physiol., 37: 263-324.

[29] F. C. Dyer (2002). The biology of the dance language. Ann Rev Entomol, 47: 917-949.

[30] M. J. Couvillon (2012). The dance legacy of Karl von Frisch. Insectes Soc., 59: 297-306. doi: $10.1007 / \mathrm{s} 00040-012-0224-\mathrm{Z}$

[31] J. C. Biesmeijer and T. D. Seeley (2005). The use of waggle dance information by honey bees throughout their foraging careers. Behav. Ecol. Sociobiol., 59: 133-142.

[32] C. Grüter and W. M. Farina (2009). The honeybee waggle dance: can we follow the steps? Trends Ecol. Evol., 24: 242-247.

[33] O. Afik, A. Dagt and S. Shafir (2008). Honeybee, Apis mellifera, round dance is influenced by trace components of floral nectar. Anim. Behav., 75: 371-377. doi:10.1016/j.anbehav.2007.04.012

[34] K. D. Waddington and W. H. Kirchner (2010). Acoustical and Behavioral correlates of profitability of food sources in honey bee round dances. Ethology, 92: 1-6.

[35] K. Waddington (1982). Honey bee foraging profitability and round dance correlates. J. Comp. Physiol., 148: $297-301$.

[36] F. C. Dyer (1987). Memory and sun compensation by honey bees. J. Comp. Physiol., 160: 621-633.

[37] F. C. Dyer (1991). Bees acquire route based memories but not cognitive maps in a familiar landscape. Anim. Behav., 41: 239-246.

[38] F. C. Dyer (1991). Bees acquire route based memories but not cognitive maps in a familiar landscape. Anim. Behav., 41: 239-246.

[39] D. E. Schmitt and H. E. Esch (1993). Magnetic orientation of honeybees in the laboratory. Naturwissenschaften, 80: 41-43.

[40] H. Esch and J. Burns (1996). Distance estimation by foraging honey bees. J Exp. Biol., 199: 155-162.

[41] M. V. Srinivasan, S. W. Zhang, M. Lehrer and T. S. Collett (1996). Honeybee navigation en route to the goal: Visual flight control and odometry. J. Exp. Biol., 199: 237-244.

[42] A. Si, M. V. Srinivasan and S. Zhang (2003). Honeybee navigation: properties of the visually driven 'odometer'. J. Exp. Biol., 206: 1265-1273.

[43] M. Dacke and M. V. Srinivasan (2008). Two odometers in honeybees? J. Exp. Biol., 211: 3281- 3286.

[44] C. Grüter, E. Leadbeater and F. L. W. Ratnieks (2010). Social learning: the importance of copying others. Curr. Biol., 20: R683-R685.

[45] J. S. Pettis and T. Pankiw (1998). Grooming behavior by Apis mellifera L. in the presence of Acarapis woodi (Rennie) (Acari: Tarsonemidae). Apidologie, 29: 241-253.

[46] E. Guzman-Novoa, B. Emsen, P. Unger, L. G. Espinosa-Montaño and T. Petukhova (2012). Genotypic variability and relationships between mite infestation levels, mite damage, grooming intensity, and removal of Varroa destructor mites in selected strains of worker honey bees (Apis mellifera L.). J. Invertebr. Pathol., 110: 314-320.

[47] D. J. Pritchard (2016). Grooming by honey bees as a component of varroa resistant behavior. J. Apic. Res., 55: 38-48. 
[48] B. B. Land and T. D. Seeley (2004). The grooming invitation dance of the honey bee. Ethology, 110: 110.

[49] T. D. Seeley (1992). The tremble dance of the honey bee: message and meanings. Behav. Ecol. Sociobiol., 31: 375-383.

[50] C. Thom (2003). The tremble dance of honey bees can be caused by hive-external foraging experience. J. Exp. Biol., 206: 2111-2116.

[51] T. D. Seeley, S. Kühnholz and A. Weidenmüller (1996). The honey bee's tremble dance stimulates additional bees to function as nectar receivers. Behav. Ecol. Sociobiol., 39: 419-427.

[52] A. Stabentheiner and K. Hagmuller (1991). Sweet food means 'Hot Dancing' in honeybees. Naturwissenschaften, 78: 471-473.

[53] K. Rohrseitz and J. Tautz (1999). Honeybee dance communications: waggle run direction coded in antennal contacts? J. Comp. Physiol. A, 184: 463-470.

[54] A. Michelsen, W. H. Kirchner and M. Lindauer (1986). Sound and vibrational signals in the dance language of the honeybee, Apis mellifera. Behav. Ecol. Sociobiol., 18: 207-212.

[55] A. Michelsen, W. F. Towne, W. H. Kirchner and P. Kryger (1987). The acoustic near field of a dancing honeybee. J. Comp. Physiol. A, 161: 633-643.

[56] W. F. Towne and W. H. Kirchner (1989). Hearing in honey bees: detection of air-particle oscillations. Science, 244: 686-688.

[57] W. H. Kirchner, C. Dreller and W. F. Towne (1991). Hearing in honeybees: operant conditioning and spontaneous reactions to airborne sound. J. Comp. Physiol. A, 168: 85-89.

[58] C. Dreller and W. H. Kirchner (1993). Hearing in honeybees: localization of the auditory sense organ. J. Comp. Physiol, 173: 275-279.

[59] J. Tautz, J. Casas and D. Sandeman (2001). Phase reversal of vibratory signals in honeycomb may assist dancing honeybees to attract their audience. J. Exp. Biol., 204: 3737-3746.

[60] O. Kilpinen and J. Storm (1997). Biophysics of the subgenual organ of the honeybee, Apis mellifera. J. Comp. Physiol A, 181: 309-318.

[61] Y. Hasegawa and H. Ikeno (2011). How do honey bees attract nestmates using waggle dances in dark and noisy hives? PLoS One, 6: e19619. doi: 10.1371/journal.pone.0019619 